\title{
RELATION BETWEEN STELLAR MASS AND STAR-FORMATION ACTIVITY IN GALAXIES*
}

\author{
Bahram Mobasher ${ }^{1}$, Tomas Dahlen ${ }^{2}$, Andrew Hopkins ${ }^{3}$, Nick Z. Scoville ${ }^{4}$, Peter Capak ${ }^{4}$, R. Michael Rich ${ }^{5}$, \\ David B. Sanders ${ }^{6}$, Eva Schinnerer 7 , Olivier Ilbert ${ }^{6}$, Mara Salvato ${ }^{4}$, and Kartik Sheth $^{8}$ \\ ${ }^{1}$ Department of Physics and Astronomy, University of California, Riverside, CA 92521, USA \\ ${ }^{2}$ Space Telescope Science Institute, 3700 San Martin Drive, Baltimore, MD 21218, USA \\ ${ }^{3}$ School of Physics, University of Sydney, NSW 2006, Australia \\ ${ }^{4}$ California Institute of Technology, MC 105-24, 1200 East California Boulevard, Pasadena, CA 91125, USA \\ ${ }_{5}$ Department of Physics and Astronomy, University of California, Los Angles, CA 90095, USA \\ ${ }^{6}$ Institute for Astronomy, 2680 Woodlawn Dr., University of Hawaii, Honolulu, HI 96822, USA \\ ${ }^{7}$ Max Planck Institute for Astronomy, Konigstuhl 17, 69117 Heidelberg, Germany \\ ${ }^{8}$ Spitzer Science Center, California Institute of Technology, Pasadena, CA 91125, USA \\ Received 2008 April 8; accepted 2008 August 18; published 2008 December 2
}

\begin{abstract}
For a mass-selected sample of 66544 galaxies with photometric redshifts $\left(z_{\text {phot }}\right)$ from the Cosmic Evolution Survey (COSMOS), we examine the evolution of star-formation activity as a function of stellar mass in galaxies. We estimate the cosmic star-formation rates (SFRs) over the range $0.2<z_{\text {phot }}<1.2$, using the rest-frame $2800 \AA$ flux (corrected for extinction). We find the mean SFR to be a strong function of the galactic stellar mass at any given redshift, with massive systems $\left(\log \left(M / M_{\odot}\right)>10.5\right)$ contributing less (by a factor of $\left.\sim 5\right)$ to the total star-formation rate density (SFRD). Combining data from the COSMOS and Gemini Deep Deep Survey, we extend the SFRD- $z$ relation as a function of stellar mass to $z \sim 2$. For massive galaxies, we find a steep increase in the SFRD $-z$ relation to $z \sim 2$; for the less-massive systems, the SFRD which also increases from $z=0$ to 1 levels off at $z \sim 1$. This implies that the massive systems have had their major star-formation activity at earlier epochs $(z>2)$ than the lower-mass galaxies. We study changes in the SFRDs as a function of both redshift and stellar mass for galaxies of different spectral types. We find that the slope of the SFRD- $z$ relation for different spectral types of galaxies is a strong function of their stellar mass. For low- and intermediate-mass systems, the main contribution to the cosmic SFRD comes from the star-forming galaxies while, for more-massive systems, the evolved galaxies are the most dominant population.
\end{abstract}

Key words: galaxies: evolution - galaxies: starburst

Online-only material: color figure

\section{INTRODUCTION}

Among the most outstanding issues in observational astronomy today is understanding the physics of the star-formation process and the parameters responsible for it. The cosmic evolution of the star-formation rate (SFR), for example, provides clues toward the assembly of mass and the development of the Hubble sequence, while parameters which govern the starformation activity are directly responsible for the evolution of galaxies. Despite significant progress on this subject in recent years, there are still a number of open questions concerning the star-formation process in galaxies.

The main problems in studying star formation in galaxies can be divided into two general groups: first, uncertainties due

\footnotetext{
* Based on observations with the NASA/ESA Hubble Space Telescope, obtained at the Space Telescope Science Institute, which is operated by AURA Inc., under NASA contract NAS 5-26555; also based on data collected at the Subaru Telescope, which is operated by the National Astronomical Observatory of Japan; the XMM-Newton, an ESA science mission with instruments and contributions directly funded by ESA Member States and NASA; the European Southern Observatory under Large Program 175.A-0839, Chile; Kitt Peak National Observatory, Cerro Tololo Inter-American Observatory, and the National Optical Astronomy Observatory, which are operated by the Association of Universities for Research in Astronomy, Inc. (AURA), under cooperative agreement with the National Science Foundation; the National Radio Astronomy Observatory which is a facility of the National Science Foundation operated under cooperative agreement by Associated Universities, Inc.; and the Canada-France-Hawaii Telescope with MegaPrime/MegaCam operated as a joint project by the CFHT Corporation, CEA/DAPNIA, the National Research Council of Canada, the Canadian Astronomy Data Centre, the Centre National de la Recherche Scientifique de France, TERAPIX and the University of Hawaii.
}

to dust extinction and variation in the properties of different star-formation diagnostics (Cram et al. 1998; Hopkins et al. 2001; Sullivan et al. 2001); second, lack of knowledge about the parameters which control star-formation activity in galaxies (Ellison et al. 2007; Li et al. 2007; Kaufmann et al. 2007; Gómez et al. 2003). There have been major advances in resolving the problems in the first group by comparing SFR estimates from different diagnostics (Cram et al. 1998; Hopkins et al. 2003; Hopkins 2004; Daddi et al. 2007), investigating the cause of their difference (Sullivan et al. 2001), and exploring the role of dust extinction in SFR measurements (Calzetti et al. 2007; Sullivan et al. 2001). Due to severe observational constraints, however, there has been little progress in addressing problems in the second group.

Cowie et al. (1996) first studied the dependence of the specific SFR (SFR per unit mass) on redshift. They found the starforming galaxies to have relatively brighter absolute $K$-band luminosities $\left(M_{K}\right)$ at higher redshifts $(z \sim 1)$ compared to those at lower $z$. As the $M_{K}$ is analogous to the integrated stellar mass of galaxies, they conclude that higher-mass systems mainly contribute to the star-formation rate density (SFRD) at higher redshifts with the main contribution shifting to lower-mass systems at lower $z$, a phenomenon they called "downsizing." This was confirmed in later studies of nearby galaxies (Brinchmann et al. 2004; Heavens et al. 2004) and more distant systems (Juneau et al. 2005; Zheng et al. 2007; Scoville et al. 2007b). Observed changes in "downsizing" as a function of redshift reveals that the most-massive galaxies form first, with galaxy formation proceeding later on to smaller systems (Juneau et al. 2005). 
The correlation between stellar mass and SFR in galaxies as a function of redshift provides insights into the integrated SFR over the history of galaxies, and hence a picture of galaxy evolution over cosmic time (Papovich et al. 2006; Dickinson et al. 2004). Moreover, the study of these relations for different morphological types of galaxies elucidates the origin of the Hubble sequence (Abraham et al. 2007; Scarlata et al. 2007).

Here, we study the relation between SFR with stellar mass and redshift, using a homogeneous, complete and unbiased sample of galaxies covering a wide range in all these parameters. We use the extensive multiwavelength data from the Cosmic Evolution Survey (COSMOS; Scoville et al. 2007a, Mobasher et al. 2007; Capak et al. 2007a). The combination of the depth, area, and wavelength coverage in COSMOS is extremely beneficial for such investigations and complements existing deeper but smaller area surveys (Juneau et al. 2005; Glazebrook et al. 2005; Giavalisco et al. 2004). Results from this study are extensively used in Scoville et al. (2007b) to explore the influence of local density enhancement on star-formation activity in galaxies.

Throughout this paper, we assume $H_{0}=70 \mathrm{~km} \mathrm{~s}^{-1} \mathrm{Mpc}^{-1}$, $\Omega_{\Lambda}=0.70$, and $\Omega_{M}=0.30$. Magnitudes are given in the $\mathrm{AB}$ system.

\section{OBSERVATIONS, PHOTOMETRIC REDSHIFTS, AND STELLAR MASSES}

We use the photometric data obtained from our imaging surveys of the COSMOS field. This includes data in $U$ (CanadaFrance-Hawaii Telescope (CFHT)), BVriz (Subaru), and $K_{s}$ (Kitt Peak/Cerro Tololo Inter-American Observatory (CTIO)) bands, covering the entire $2 \mathrm{deg}^{2}$ area of the COSMOS field. Details of the photometric observations and data reduction are presented in Capak et al. (2007a) and Taniguchi et al. (2007). For the present study, we use a subset of the $i$-band-selected catalog, extended over the entire COSMOS field and complete to $i<25 \mathrm{mag}$. To this magnitude limit, we derive accurate photometric redshifts and spectral types (Mobasher et al. 2007), and can reliably estimate the SFRs. This covers a significantly larger area with a depth similar to previous studies (see the compilation by Hopkins \& Beacom 2006).

Photometric redshifts for galaxies in COSMOS are calculated using the template fitting method incorporating priors, as described in Mobasher et al. (2007). For each object, we derive the photometric redshift, the redshift probability distribution, and the best-fitting spectral type. The spectral types cover E, Sbc, Scd, and Im templates (Coleman et al. 1980, extended to the UV and NIR by Bolzonella et al. 2000) in addition to two starburst templates (Kinney et al. 1996). We also consider extinction as an independent variable in the spectral energy distribution (SED) fits.

It is possible that by considering extinction as a free parameter in the fit, we increase the likelihood of degeneracy in the final result (i.e., redshift, dust extinction, and the presence of the old population all make the SEDs redder). This is examined in Mobasher et al. (2007) by studying the accuracy of photometric redshifts by comparing the photometric $\left(z_{\text {phot }}\right)$ and spectroscopic $\left(z_{\mathrm{spec}}\right)$ redshifts for a sample of galaxies in the COSMOS with such available data, expressed by the ratio $\Delta_{z} \equiv\left\langle\left|z_{\text {phot }}-z_{\text {spec }}\right| /\left(1+z_{\text {spec }}\right)\right\rangle$. The smallest rms scatter between the estimated photometric and available spectroscopic redshifts is obtained when treating the extinction as a free parameter (see Table 4 and Figure 5 in Mobasher et al. 2007). We find $\Delta_{z}=0.03$ with a small fraction $(\sim 2.5 \%)$ of outliers, defined as $\Delta_{z}>0.3$. Since Mobasher et al. (2007) use a subsample of the COSMOS galaxies with available spectroscopy to calibrate photometric redshifts, it is possible that the photometric redshift sample is biased against fainter galaxies, for which spectroscopic data do not exist in COSMOS. To minimize this, we constrain the sample to galaxies with $z<1.10$ as these have the most reliable photometric redshifts and smallest number of outliers (Mobasher et al. 2007).

We estimate the stellar mass corresponding to each galaxy, using the photometric redshift, the rest-frame $V$-band absolute magnitudes $\left(M_{V}\right)$, and the $M / L_{V}$ ratio dependent on the spectral type estimated from the best-fit SED for each galaxy, as described in Mobasher et al. (2007).

\section{STAR-FORMATION RATES FROM UV LUMINOSITIES}

The SFRs associated with individual galaxies are estimated using rest-frame $2800 \AA$ Aluxes. We estimate this for each galaxy, using photometric data covering $U B V r i z K$ bands. This is done by interpolating between the two bands that straddle the restframe $2800 \AA$ wavelength, making use of the shape of the best-fit SED when doing the interpolation. The exception is at low redshifts $(0.20<z<0.34)$, where we use the observed $U$-band magnitude, extrapolated to $2800 \AA$ using the bestfit SED. Having the observed apparent flux, $K$-correction, and photometric redshifts obtained from the best-fit SEDs, we then estimate the absolute flux in rest-frame $\lambda=2800 \AA$. A detailed description of this technique is presented in Dahlen et al. (2007).

The UV luminosity at $2800 \AA$ is mainly produced by shortlived $\mathrm{O}$ and $\mathrm{B}$ stars and is therefore closely related to the ongoing star-formation activity. Here, we follow the approach in Dahlen et al. (2007) to relate the UV flux to the SFR. This involves using predictions from stellar synthesis models and assuming parametric forms for the past star-formation history to mimic the evolution of the SFR with redshift, as summarized below. We derive the conversion factor between the UV flux and the ongoing SFR, using the stellar population synthesis code GALEXEV (Bruzual \& Charlot 2003). We assume solar metallicity and a modified Salpeter initial mass function (IMF) spanning $0.1<M / M_{\odot}<100$, but having a turnover at low mass (Baldry \& Glazebrook 2003). To model the dependence of SFR on redshift, we use a parametric fit to the data from the Great Observatories Origins Deep Survey (GOODS), presented in Giavalisco et al. (2004) and parameterized in Strolger et al. (2004). Convolving the past SFR history with the results from the stellar synthesis models, we derive a redshift-dependent conversion factor, $k_{\lambda}(z)$, between the ongoing SFR and UV flux as

$$
L_{2800}=k_{2800}(z) \frac{\mathrm{SFR}}{M_{\odot} \mathrm{yr}^{-1}} \mathrm{ergs}^{-1} \mathrm{~Hz}^{-1} .
$$

The dependence of $k_{2800}(z)$ on redshift is shown in Figure 1. Note that we use the same volume-averaged past SFR history to calculate the conversion factor for all galaxies. Therefore, galaxies that have SFR histories significantly different from the average may be assigned an SFR that differs from the actual value. Furthermore, galaxies of different stellar masses are characterized by different star-formation histories (Panter et al. 2007), introducing a mass-dependent bias into the SFR estimates for individual galaxies. We do not correct the SFR calibration here for this effect, as this is highly model dependent and introduces more free parameters into the analysis. Moreover, any such correction would complicate the interpretation of a mass-SFRredshift relation, discussed in the following sections. We show 


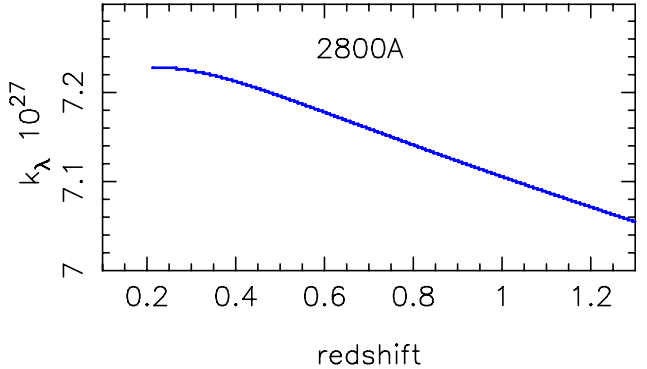

Figure 1. Plot of the conversion factor, $k_{2800}$, between UV luminosity (at $2800 \AA$ ) and ongoing SFR as a function of redshift. $L_{2800}=k_{2800}(z) \frac{\mathrm{SFR}}{M_{\odot} \mathrm{yr}^{-1}}$ erg s${ }^{-1} \mathrm{~Hz}^{-1}$. The star-formation history used is taken from Strolger et al. (2004), while stellar synthesis models are taken from Bruzual \& Charlot (2003), assuming solar metallicity and a Salpeter IMF. The conversion factor is given in units of $10^{27}$

(A color version of this figure is available in the online journal.)

the redshift dependence of the conversion factor in Figure 1, illustrating only a weak dependence with a change of only $2 \%$ over the redshift interval considered here. This suggests that the dependence on the assumed SFR history is weak when deriving the SFR. While this is an acknowledged source of uncertainty affecting calibration of the SFR for any individual galaxy, it has no effect on the estimates of the total SFRD. This is because the SFRD is calculated by integrating over UV luminosity functions (LFs) estimated from the data, after scaling the UV luminosity appropriately to SFR using the redshift-dependent conversion shown in Figure 1. We will return to the discussion of the accuracy of the SFR calibration again in Section 5.1, when we compare our estimated SFRDs with those derived independently and compiled from the literature.

Accurate estimate of dust extinction is essential in correcting the SFRs measured from the rest-frame UV (2800 ̊) flux. Using the extinction values estimated for individual galaxies through the SED fits, and assuming a Calzetti extinction law (Calzetti et al. 2000), we derive the reddening, $E_{B-V}$, for each galaxy. To examine the accuracy of the $E_{B-V}$ values in this study, we explore their distribution for different spectral types of galaxies. As expected, we find the median extinction $\left(E_{B-V}\right)$ values to increase in galaxies from old and evolved to young and star-forming spectral types. Furthermore, we estimate the dust-corrected SFRDs as presented in Section 5.1, with different extinction corrections: the median $E_{B-V}$ values for each spectral type, individual extinction corrections for each galaxy, and a constant $E_{B-V}$ value for all the galaxies. We find that the final results do not depend on the specific prescription for extinction correction.

\section{SAMPLE SELECTION AND COMPLETENESS}

The sample considered in the present study is selected in the $i$ band. This is the filter with the deepest COSMOS photometry and provides a sample most like a mass-selected survey (at least to $z \sim 0.5$ ). Samples of star-forming galaxies, selected based on emission lines ([O II], $\mathrm{H} \alpha$ ), broadband (i.e., UV, $U$ band), and far-infrared (IR) or radio flux, can be very biased, since they select only a particular class of galaxies. This is the main reason for selecting a sample resembling a stellar mass-selected survey over much of its redshift range. The final sample has

1. $i_{A B}<25$ : the majority of the objects fainter than this limit escape detection at some wavelengths (especially in the NIR), resulting in poor wavelength coverage of the SEDs, and thus uncertain photometric redshifts, SFRs, and stellar masses.

2. $0.20<z<1.10$ : the lower limit for the photometric redshift is adopted to minimize extrapolation to rest-frame $2800 \AA$ for low-redshift galaxies, while the upper limit is selected to avoid objects with uncertain photometric redshifts (Mobasher et al. 2007).

3. $9.5<\log \left(M / M_{\odot}\right)<11.5$ : this allows a sample complete in terms of stellar mass. (Figure 2 shows the distribution of stellar mass as a function of redshift). We find that galaxies with redder SEDs (i.e., those with spectral types resembling the early-type systems) are highly incomplete to $\log \left(M / M_{\odot}\right) \sim 9.5$ while this is the appropriate completeness limit for galaxies with bluer SEDs (corresponding to later-type systems).

4. $0 M_{\odot} \mathrm{yr}^{-1}<\mathrm{SFR}_{2800}<100 M_{\odot} \mathrm{yr}^{-1}$ : this will exclude sources with excessive (and probably uncertain) SFRs, which could dominate the SFRDs, and active galactic nuclei (AGNs) wrongly classified as star-forming galaxies due to their extreme UV flux. Figure 3 shows the SFR distribution as a function of redshift. Very few galaxies are found to have $\mathrm{SFR}_{2800}>100 M_{\odot} \mathrm{yr}^{-1}$, and therefore the results are not sensitive to the upper limits imposed for the SFRs.

5. $M_{V}<-19$ : to allow the selection of galaxies from the same part of the LF at different redshifts and minimize luminosity- (stellar mass) dependent biases. This criterion excludes only an additional 61 galaxies on top of the above criteria, predominantly located in the middle two redshift bins.

After applying the above selection criteria, we have a total of 66,544 galaxies in our sample. Following Scoville et al. (2007b), we divide the sample into the following redshift bins: $0.20<z<0.43$ (5594 galaxies); $0.43<z<0.65$ (9608 galaxies); $0.65<z<0.88$ (22,374 galaxies); and $0.88<z<1.10$ (28,968 galaxies). In terms of stellar mass, our combined sample is complete to $M \sim 3 \times 10^{9} M_{\odot}$ over the redshift range $0.20<z<1.1$.

The fraction of the AGNs in the final sample is $<1 \%$ (a total of 1865 sources; Salvato et al. 2008), as identified by their X-ray flux. We remove all the identified AGNs from the sample. There is a possibility that the final sample is still contaminated by a small number of AGNs. However, while massive galaxies at $z \sim 2$ could host obscure AGNs (Daddi et al. 2007), the fraction of galaxies at $z<1$ with strong nuclear activity is small. It is also likely that we miss a population of highly dusty star-forming galaxies with masses above our stellar mass completeness limit. Our estimated global SFRs are therefore likely to be lower limits, although our corrections for dust obscuration to the rest-frame UV flux and the technique used to estimate the total SFRDs are expected to account for the majority of such missing systems.

\section{RESULTS}

\subsection{Measurement of the Star-Formation Densities}

The aim of this study is to explore the effect of stellar mass on the star-formation activity in galaxies and to follow its behavior as a function of look-back time. Previous such studies have either been too shallow (i.e., $2 \mathrm{dF}$ and Sloan Digital Sky Survey (SDSS); Gómez et al. 2003), or covered only small volumes with very few sources (Juneau et al. 2005), therefore suffering from cosmic variance. The present study, based on the 

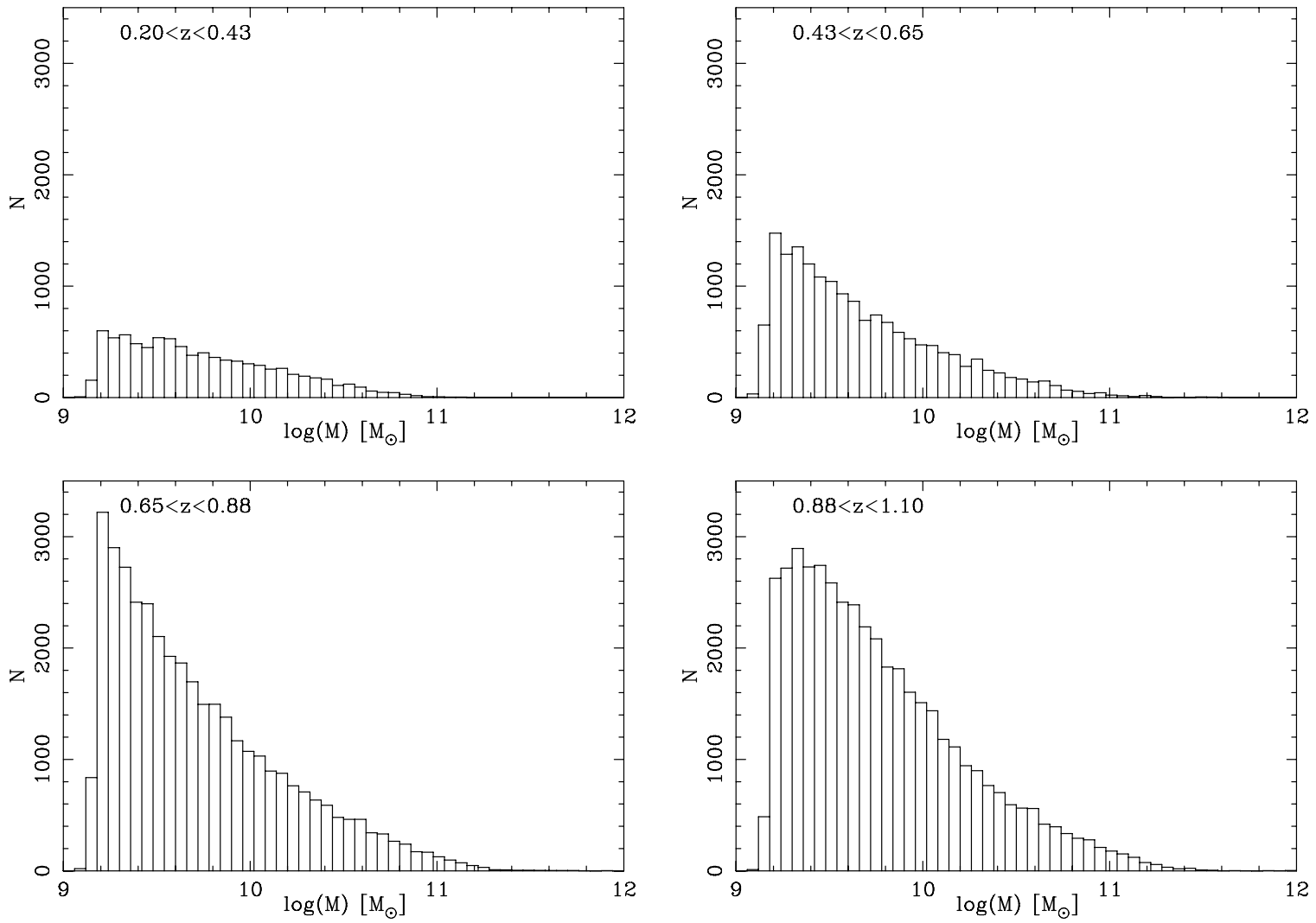

Figure 2. Distribution of the stellar mass of galaxies in different redshift intervals. The redshift bins are adopted following Scoville et al. (2007b). The mass completeness limits for the COSMOS are estimated and used to select a survey complete in stellar mass.
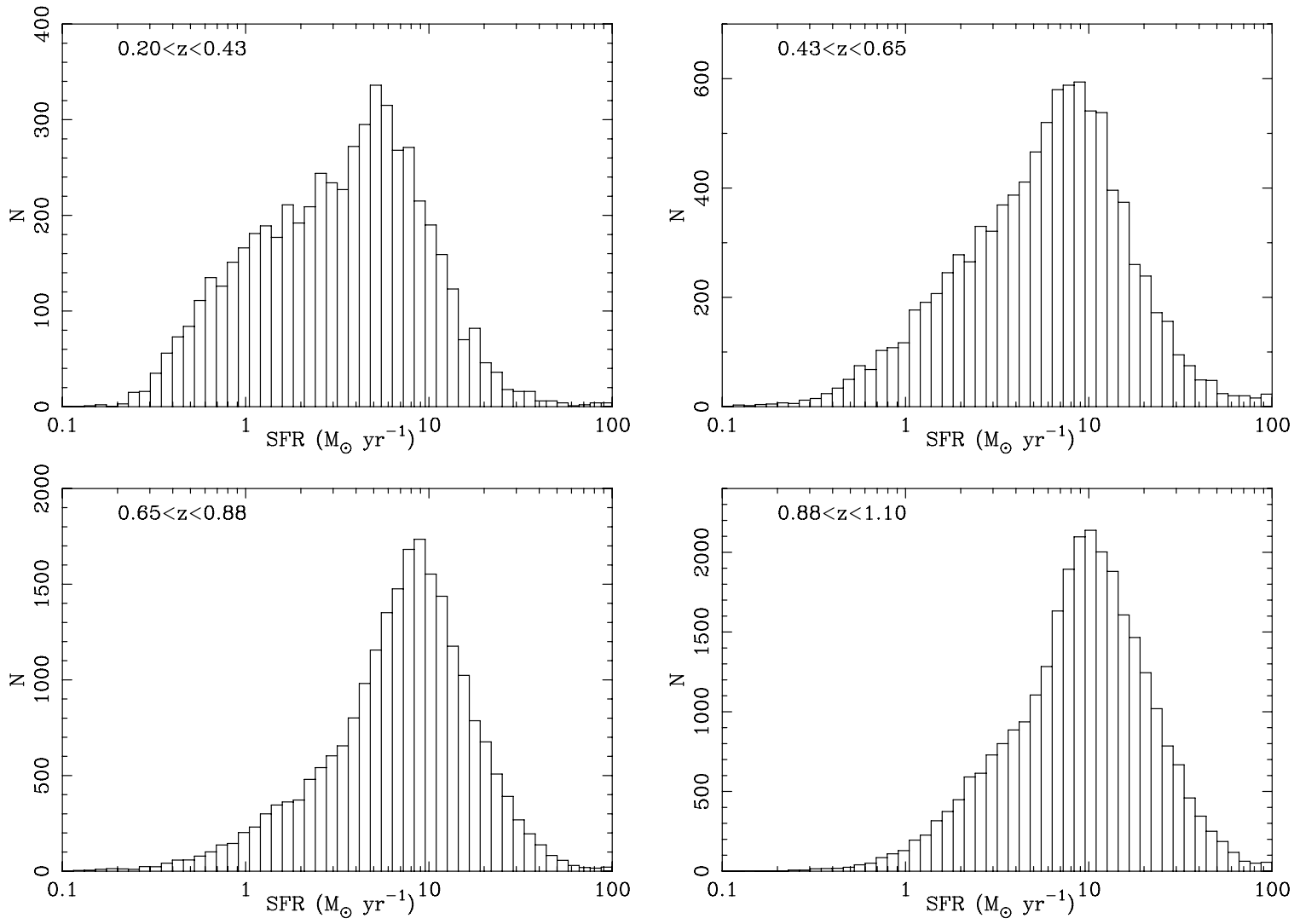

Figure 3. Distribution of the estimated SFRs (corrected for extinction) for COSMOS galaxies in different redshift intervals. 


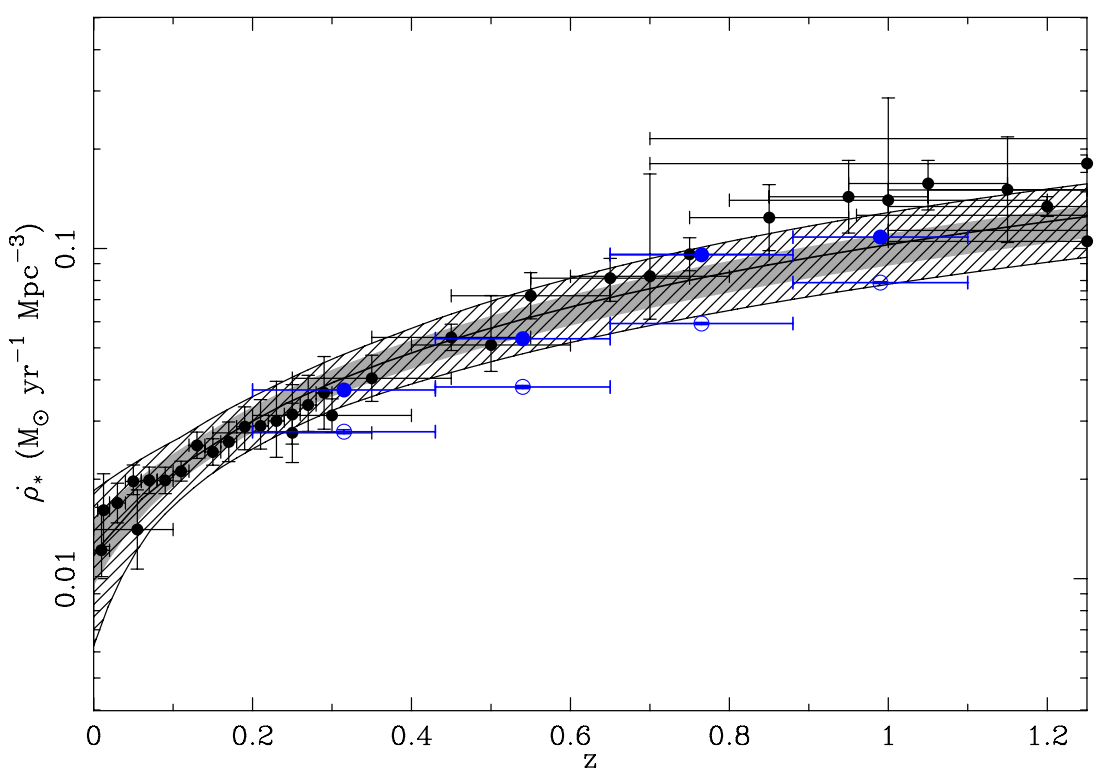

Figure 4. Extinction-corrected SFRDs, SFRD 2800 , vs. redshift, derived from COSMOS galaxies compared with other independent estimates of the, SFRD. Total SFRDs are estimated from integrating the rest-frame UV LF (filled; blue circles) or are estimated by summing over the SFRs for individual galaxies (open; blue circles). The vertical error bars correspond to Poisson counting statistics and are all smaller than the symbol size for the COSMOS galaxies (blue symbols). The filled black symbols are the compilation of data from the literature (Hopkins \& Beacom 2006), reduced to the same cosmology used here.

Table 1

SFRDs Estimated from the Sum of the SFRDs for Individual Galaxies and from Integrating the Rest-Frame LFs at Four Redshift Intervals Considered Here

\begin{tabular}{lcc}
\hline \hline$z$ & $\begin{array}{c}\log (\mathrm{SFRD})\left(M_{\odot} \mathrm{yr}^{-1} \mathrm{Mpc}^{-3}\right) \\
(\mathrm{LF})\end{array}$ & $\begin{array}{c}\log (\mathrm{SFRD})\left(M_{\odot} \mathrm{yr}^{-1} \mathrm{Mpc}^{-3}\right) \\
(\text { Simple Sum })\end{array}$ \\
\hline $0.315 \pm 0.115$ & $-1.4286 \pm 0.0058$ & $-1.5550 \pm 0.0058$ \\
$0.540 \pm 0.110$ & $-1.2733 \pm 0.0045$ & $-1.4196 \pm 0.0045$ \\
$0.765 \pm 0.115$ & $-1.0192 \pm 0.0029$ & $-1.2273 \pm 0.0029$ \\
$0.990 \pm 0.110$ & $-0.9656 \pm 0.0026$ & $-1.1033 \pm 0.0026$ \\
\hline
\end{tabular}

COSMOS field, uses data in a wide $\left(2 \mathrm{deg}^{2}\right)$ area to medium depth, providing a highly homogeneous sample in the range $0.20<z<1.10$ with well-known selection bias.

We estimate the SFRDs in four redshift intervals: $0.20<z<$ $0.43 ; 0.43<z<0.65 ; 0.65<z<0.88$; and $0.88<z<1.10$. In each redshift interval the SFRD is estimated in two ways: (1) by summing over the extinction-corrected SFRs for galaxies above the stellar mass completeness limit in each redshift interval and normalizing them to the volume corresponding to that redshift slice; (2) by integrating the rest-frame UV LF of galaxies (corrected for extinction) in the above redshift intervals, measuring the UV luminosity densities and converting them to SFRDs. The SFRDs from the two methods are listed in Table 1, with the evolution of the SFRD with redshift, derived from the COSMOS galaxies, presented in Figure 4. This is compared with the SFRDs estimated independently of other studies, as compiled in Hopkins \& Beacom (2006). All the data in Figure 4 are scaled to a modified Salpeter IMF described by Baldry \& Glazebrook (2003). Error bars are measured assuming Poisson statistics. The SFRDs per redshift bin estimated from the sum of the contributions from individual galaxies are lower than those derived from the rest-frame UV LF (Table 1 and Figure 4). This is due to the absence of sources fainter than the flux limit of the survey from this method. The agreement between the SFRDs estimated here using the extinction-corrected restframe UV LF and those in the literature over the redshift range $0.20<z<1.10$ is very good, given that the SFRDs are measured from different diagnostics, different surveys, and are based on different prescriptions for extinction correction. The agreement here lends support to our estimated values for redshift, SFR, and extinction. Because of their selection wavelengths, however, all these surveys are biased against very dusty, extremely star-forming galaxies (Afonso et al. 2003), implying that the estimated SFRDs in Figure 4 are likely to be lower limits. We further discuss this and its implications in Section 6.

\subsection{Relation Between the SFRDs and Stellar Mass}

The main result of this paper is presented in Figure 5, where we find changes in the SFRDs as a function of redshift and stellar mass. We divide the sample into three mass intervals: $9.5<\log \left(M / M_{\odot}\right)<10.0,10.0<\log \left(M / M_{\odot}\right)<10.5$, and $10.5<\log \left(M / M_{\odot}\right)<11.5$ in order to have a sufficient number of galaxies in each stellar mass interval. The points corresponding to the lower-mass bins are likely to be lower limits due to incompleteness. Error bars correspond to Poisson statistics and are often smaller than the symbol size. We find that for galaxies in all mass intervals, there is a clear increase in the SFRDs with redshift, changing by a factor of 2-4 over the redshift range considered here. Moreover, at any given redshift, more-massive galaxies $\left(\log \left(M / M_{\odot}\right)>10.5\right)$ contribute less (by a factor of 5) to the total SFRD. This is because they have already gone through their intensive star-formation phase and have built up their mass. Results from Figure 5 also imply that, while massive galaxies continue to undergo modest star-formation activity to $z \sim 1$, they have acquired most of their mass before this redshift (i.e., $z>1$ ). The results here are consistent with the "downsizing" picture of galaxy formation, where the SFR changes from high-mass to low-mass systems (Cowie et al. 1996; Juneau et al. 2005).

The mass-dependent evolution of the SFRDs show progressively smaller contributions to the total SFRD (including all masses) as the SFRD declines to low $z$. The fraction of the total SFRD, however, is more or less constant over the redshift range 


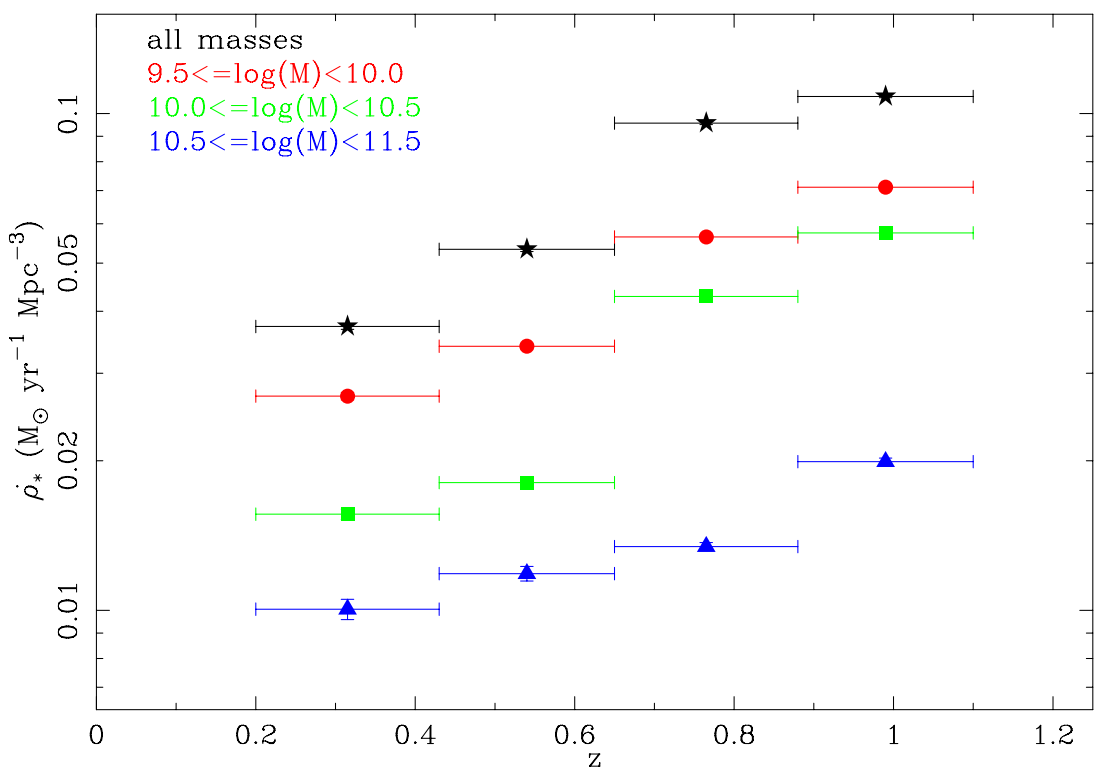

Figure 5. Extinction-corrected $\mathrm{SFR}_{2800}$ density from the COSMOS plotted against redshift in different stellar mass intervals. The error bars are again estimated assuming Poisson statistics and are typically smaller than the symbol size.

probed for each mass bin (i.e., at any given mass bin, the ratio of the SFRD in that bin to the total SFRD does not change over the redshift range considered here). This result differs significantly from that found by Seymour et al. (2008) for the evolution in the high-luminosity end of the LF, where very strong massdependent evolution is seen for this fractional contribution to the total SFRD. This result is likely to be due to the different region of the LF sampled by different selection effects arising at different wavelengths. Furthermore, the radio-selected sample used in Seymour et al. (2008) is mainly dominated by galaxies from the high-end of the LF while the optically selected sample here is more typically dominated by $L^{*}$ galaxies.

Using a sample of galaxies detected at $24 \mu \mathrm{m}$ wavelength, Zheng et al. (2007) studied the evolution of the SFRD with redshift as a function of stellar mass. Their sample covers the same redshift range as the present study and uses a "dustfree" measure of the SFR by combining the UV and the total IR flux $(8-1000 \mu \mathrm{m})$ for individual galaxies. Therefore, by comparing our results with those in Zheng et al. (2007), one could quantify possible dust-induced biases in Figure 5, despite the very different selection criteria used for these samples. The results from the two studies are compared in Figure 6, where they are divided into the same mass intervals. Over the redshift range covered by these studies, the rate of change of the SFRDs with redshift (i.e., the slope of the SFRD$z$ relations) is the same, independent of the stellar mass. For the intermediate-mass galaxies with $10^{10.19}<M / M_{\odot}<10^{11.19}$, there is excellent agreement between the two studies. However, for the lowest-mass bin at $10^{9.5}<M / M_{\odot}<10^{10.19}$, the SFRD estimated here is significantly higher (by a factor of 5) at all redshifts. This discrepancy is not caused by dust extinction in our estimate of the SFRD compared to Zheng et al. (2007), as this would have led to a relatively smaller SFRD (with respect to Zheng et al.) for our sample. This is likely to be a consequence of both incompleteness and selection effects in the lowest-mass bin of Zheng et al. (2007). Their method of IR $(24 \mu \mathrm{m})$ stacking, based on the optically selected sample of COMBO-17 sources in this bin, is biased against optically faint low-mass galaxies. These are exactly those systems that are likely (by virtue of dust obscuration) to be, on average, brighter in the IR wavelengths than that measured by the stacking result from the optically detected sources. This causes a bias, leading to an underestimation in the inferred SFRD for their lowestmass bin. Moreover, the inclusion of low-mass red galaxies in the stacking analysis (as done by Zheng et al. 2007) would serve to reduce the inferred average SFR, adding another bias toward underestimating the true SFRD. A detailed investigation of the extent of the bias in the low-mass bin of Zheng et al. (2007) study would be facilitated by discriminating between blue (starforming) and red (non-star-forming) populations. Given the above discussion, it is likely that their result for the lowestmass bin is an underestimate. The disagreement seen in the lowest-mass bin in Figure 6 is therefore not surprising. To summarize, while for the intermediate-mass galaxies the SFRDs at different redshifts are in excellent agreement, the observed discrepancy for the lowest-mass galaxies is likely to be caused by incompleteness and selection bias in the Zheng et al. sample. Correcting for these effects brings Zheng et al.'s result into better agreement with that in the present study. For the highest-mass bin $\left(M>10^{11.15} M_{\odot}\right)$, there is serious incompleteness in our sample, which explains the relatively lower SFRDs measured for galaxies with higher stellar mass from the present study.

We find the number density evolution in the present sample to depend on the stellar mass of galaxies. The impact of this number density evolution on the SFRD can be interpreted by considering the ratio of the SFRD $\left(\dot{\rho}_{*}\right)$ to the number density of star-forming galaxies $\left(\rho_{N}\right), \dot{\rho}_{*} / \rho_{N}$, defined as the "characteristic star-formation rate" (cSFR), as a function of mass and redshift. The cSFR remains flat for our low-mass galaxies, at a level of $\approx 1 M_{\odot} \mathrm{yr}^{-1}$, while dropping by more than an order of magnitude for high-mass systems from $\approx 2 M_{\odot} \mathrm{yr}^{-1}$ at $z \approx 1$ to $\approx 0.1 M_{\odot} \mathrm{yr}^{-1}$ at $z \approx 0.3$. Intermediate masses show an intermediate level of evolution, with their cSFRs comparable to the high-mass objects at high $z$ and to low-mass objects at low $z$. This behavior exhibits the characteristics of "downsizing" in galaxy evolution in the sense that the cSFR decreases with redshift faster for massive galaxies than for low-mass systems. 


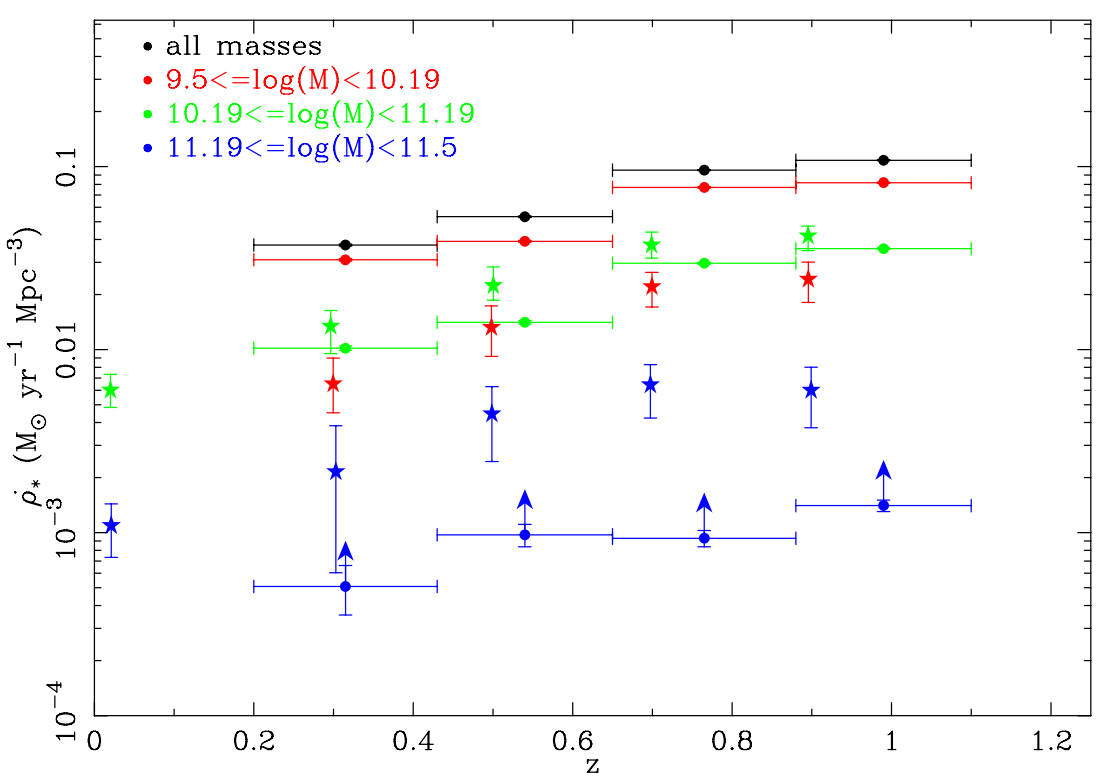

Figure 6. SFRDs from the COSMOS (this study) (filled dots) compared with those from Zheng et al. (2007) (stars). Different colors indicate different mass intervals. The two samples are binned in the same stellar mass intervals. The SFRDs from Zheng et al. (2007) are estimated using the combined UV and total IR (8-1000 $\mu \mathrm{m})$ flux of galaxies. The error bars correspond to Poisson statistics.

Using spectroscopic observations of galaxies over the range $1<z<2$, selected from the Gemini Deep Deep Survey (GDDS), Juneau et al. (2005) studied changes in the SFRDs with redshift as a function of stellar mass of galaxies. This provides a natural extension of the COSMOS study to $z \sim 2$. To study the behavior of this relation over the redshift range covered by the combined COSMOS and GDDS, we divide our sample into the same mass bins as those in Juneau et al. (2005) and compare the results in Figure 7. For the most-massive galaxies we find the trend in SFRD with redshift to continue to $z \sim 2$, however for the less-massive systems it flattens around $z \sim 1.1$. This implies that downsizing has been effective at $z>1$, and that the massive galaxies were formed before $z \sim 2$ after going through a period of intensive star-formation activity. Results in Figures 5 and 7 confirm that, at any given redshift out to $z \sim 2$, massive galaxies contribute less to the total SFRDs than objects with smaller stellar mass. However, although the sample here is selected to be close to Juneau et al.'s galaxies (in terms of the range in their stellar mass), differences in the selection criteria between the two samples are likely to affect the above result.

\subsection{Evolution of the SFRD as a Function of Stellar Mass and Galaxy Type}

The coverage of the COSMOS field by the Advanced Camera for Surveys (ACS), on board the Hubble Space Telescope (HST), provides high-resolution imaging of galaxies and measurement of their morphologies. Since the coverage is in only one $H S T$ /ACS band, it is not possible to measure morphologies for galaxies at the same rest-frame wavelength in all the four redshift bins studied here. We therefore use the spectral types as a proxy to rest-frame ACS morphologies. We have already shown that our estimated spectral types closely agree with the rest-frame morphologies in COSMOS (Capak et al. 2007b). Nevertheless, one needs to be cautious that the spectral types and morphologies of galaxies are only loosely related. In the following discussion, these terms are used synonymously. Moreover, relations involving photometric redshifts, spectral types, and stellar masses of galaxies should be interpreted carefully as these parameters are not independently derived here.

In Figure 8, we present the evolution of the SFRD with redshift as a function of both stellar mass and spectral type of galaxies. The spectral types are divided into those with redder SEDs (early types), intermediate-color SEDs (spirals), and bluer SEDs (late types and starbursts; Mobasher et al. 2007). As with any type classification, there will be some level of misclassification or systems that do not fall easily into the defined spectral types. For the following discussion, this limitation must be kept in mind. In particular, there is some ambiguity regarding irregular galaxies, which may in some instances fall either in the "spiral" or "starburst" classes as all these are represented by SEDs similar to those of star-forming galaxies. Nevertheless, we find a clear trend for all the spectral types in Figure 8. Due to serious incompleteness in galaxies with redder SEDs at $\log \left(M / M_{\odot}\right) \sim 9.5$, the relation for these galaxies is not shown in Figure 8. For the low- and intermediatemass galaxies, starbursts dominate the star-formation activity at high redshifts with a comparable contribution from spirals at lower redshifts. Also, for the lowest-mass systems $(9.5<$ $\left.\log \left(M / M_{\odot}\right)<10\right)$, we find a steep increase with redshift in the SFRD for starbursts, compared to that for the spirals which remain unchanged with redshift. However, for the intermediatemass systems $\left(10<\log \left(M / M_{\odot}\right)<10.5\right)$, the slope of the SFRD $-z$ relation increases for both spirals and starbursts, with the spirals making a relatively higher contribution to the total SFRD at lower redshifts. For higher-mass galaxies, the sample is dominated by early types, with the spirals showing a relatively steeper change in their SFRD with redshift compared to earlier types. This is consistent with the expectation from the downsizing scenario that the most-massive systems are dominated by early-type old galaxies, which have undergone intense star-formation activity at higher redshifts $(z \approx 1)$. An interesting feature in Figure 7 is that the slopes of the SFRD$z$ relation for different spectral types of galaxies are strong functions of their stellar mass.

Perhaps the most revealing population in this diagram is the starbursts. These are a dominant form of star formation 


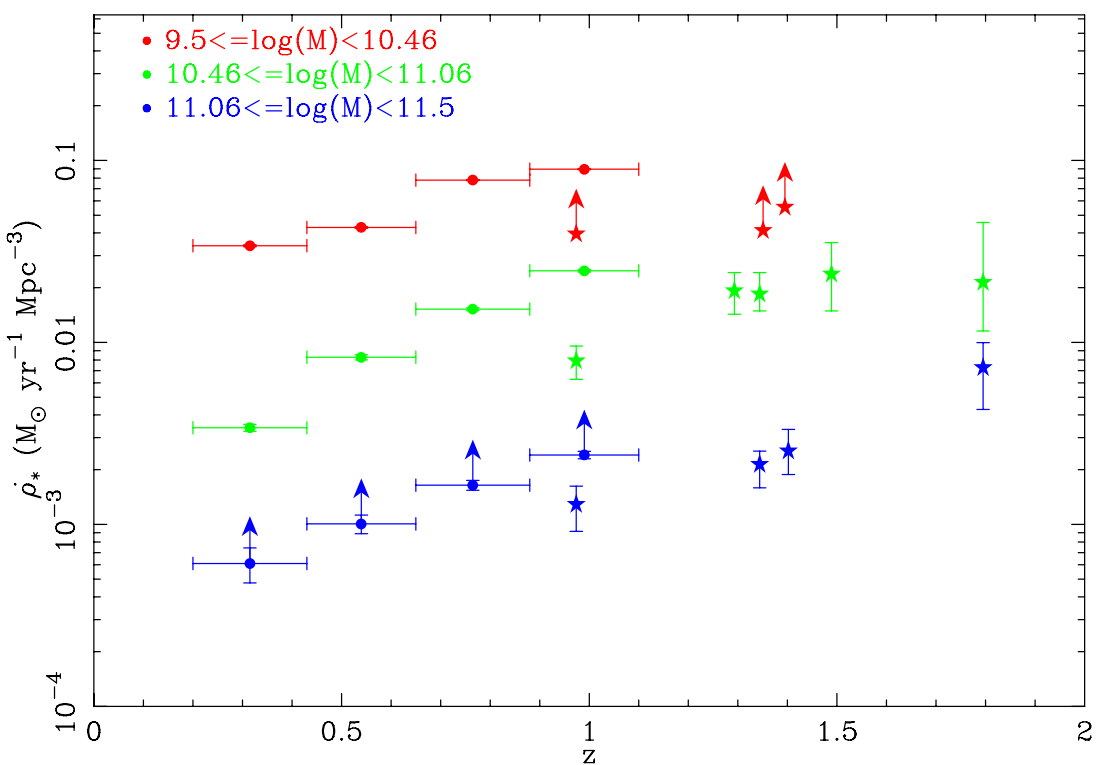

Figure 7. Changes in SFRD with redshift in different stellar mass intervals, extended to $z \sim 2$ using the Juneau et al. (2005) estimates from the GDDS (asterisk). The SFRDs from the COSMOS are re-binned to the same mass intervals as those in the Juneau et al. (2005) sample. The data from the GDDS are reduced to the same cosmology and IMF as for the COSMOS.

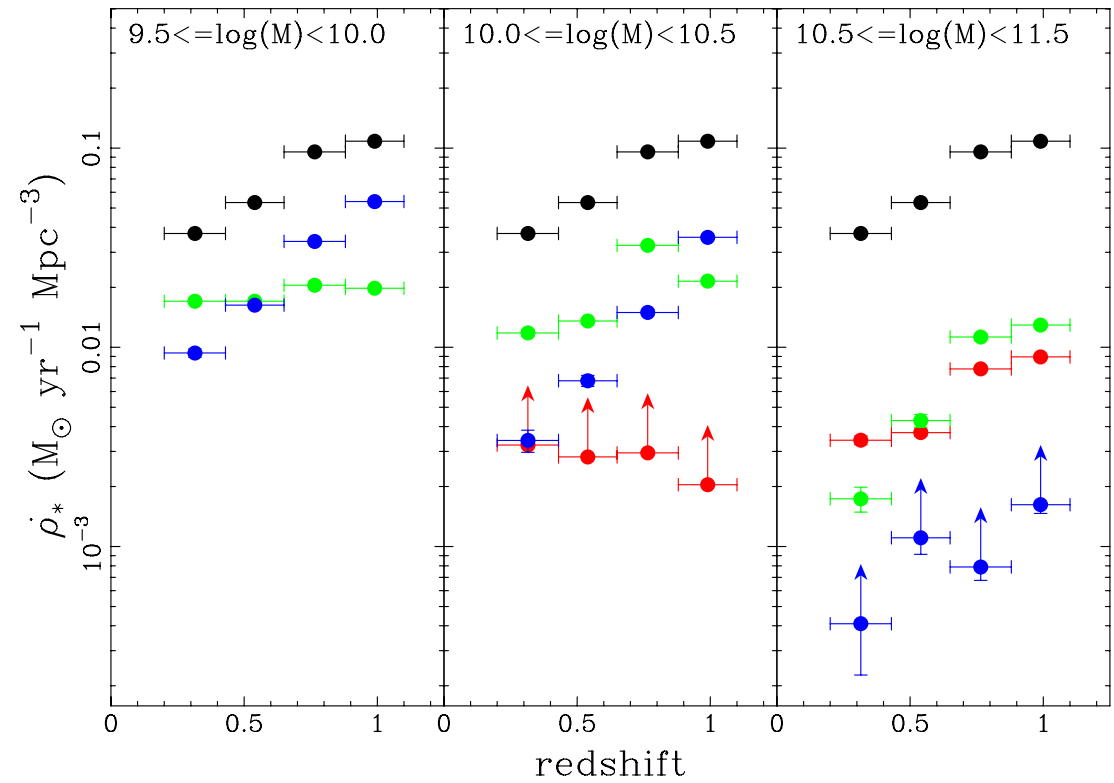

Figure 8. SFRD as a function of stellar mass and galaxy type. Blue: starburst; green: spiral (Sa-Sd); red: early (E/S0); black: all types.

in galaxies at $z \gtrsim 1$, but their prevalence at lower redshifts declines progressively with decreasing redshift. This is reflected in the sharp decrease from $z \sim 1$ to $z \sim 0.20$ of the starburst contribution to the SFRD with redshift for low- and intermediate-mass systems. The fact that most definitions of a starburst favor low-mass star-forming galaxies can be seen in the relatively large contribution of this population in the lowestmass bin, and progressively less toward higher masses. In the high-mass bin, there are too few starbursts observed to reliably fit LFs, and the lower limits shown reflect the sum over the detected population only. Overall, starbursts account for the majority of the star formation in low- to mid-mass systems at higher redshifts $(z \gtrsim 0.7)$, but at lower redshifts the dominant population shifts to the spiral types in these mass ranges. The lower the galaxy mass, the lower the redshift to which the starbursts remain dominant, consistent with the low-mass irregular starburst galaxies seen in the local universe (Figure 3 in
Kennicutt 1998). This is a consequence of the change of slope of SFRD $-z$ relations found in different stellar mass intervals.

The spiral types are the dominant contributors at low redshift and low and intermediate mass, although they make a contribution similar to the early types in the highest-mass bin. The evolution of the SFRD for spirals is flatter than any other classes, which is due to a transformation from starburst to spiral spectral types with decreasing redshift. We should note that, particularly for early types, the lowest-mass systems are expected to form their stars very late in most hierarchical models.

\section{DISCUSSION}

A number of studies in recent years have explored changes in SFRD with redshift as a function of the stellar mass of galaxies (Glazebrook et al. 2005; Juneau et al. 2005; Zheng et al. 2007). Here, we investigate this relation using a large and 
homogeneous sample of galaxies with well-known selection biases, accurate photometric redshifts, SFR estimates, and stellar mass measurements.

We find a strong dependence of the SFRD on stellar mass in the range $0.20<z<1.10$, with higher-mass galaxies $\left(M>10^{10.5} M_{\odot}\right)$ contributing less (by an order of magnitude) to the global SFRD than lower-mass systems at any given redshift. However, the rate of evolution of the SFRD with redshift is the same, regardless of the stellar mass of galaxies, in agreement with the independent study by Zheng et al. (2007), in which the effect of dust obscuration in the selection of star-forming galaxies and measurement of their SFRs is taken into account.

Using the GDDS with spectroscopic redshifts in the range $1<z<2$, Juneau et al. (2005) studied this relation from $z \sim 1$ to $z \sim 2$. The combined COSMOS and GDDS data show that for high-mass galaxies $\left(11.06<\log \left(M / M_{\odot}\right)<11.76\right)$ the upward trend in the SFRD continues to $z \sim 2$, while for lower-mass systems, after a steep increase with redshift, the SFRD flattens at $z>1$. This implies that massive galaxies produced most of their stars by $z \sim 2$ (when the universe was $\sim 3.5 \mathrm{Gyr}$ old), with the less-massive galaxies hosting efficient star-formation activity only after $z \sim 1$. These results confirm that the stellar mass of galaxies regulates the relative contribution of a galaxy to the global SFRD, consistent with the "downsizing" scenario for the formation of galaxies. Moreover, we find that galaxy "downsizing" was already occurring at $z \sim 2$ and has continued to the present epoch.

The estimated SFRD in this study is likely to be a lower limit due to selection at optical bands biasing against dusty starforming galaxies, which could significantly contribute to the global SFRD. Using a near-IR-selected sample with $K_{s}<21.5$, mimicking a mass-selected sample complete to $M \sim 10^{10} M_{\odot}$, Caputi et al. (2006) estimated the space density of luminous infrared galaxies (LIRGs) and ultra-luminous infrared galaxies (ULIRGs) in the range $0.5<z<1$, similar to the redshift range covered in the present study. They found that $24 \%$ of galaxies with $10^{11} M_{\odot}<M<2.5 \times 10^{11} M_{\odot}$ are either LIRGs or ULIRGs (excluding AGNs), with a negligible fraction of LIRGs and ULIRGs to have masses in excess of $2.5 \times 10^{11} M_{\odot}$. Furthermore, using the MIPS observations in the GOODS-S field, Caputi et al. (2005) show a very small fraction of LIRGs or ULIRGs at $0.4<z<1$ to have $M<5 \times 10^{10} M_{\odot}$. Indeed, ULIRGs of any stellar mass are found to be very rare below $z \sim 0.5$ (Flores et al. 1999; Caputi et al. 2006; Le Floc'h et al. 2005). The conclusion from these studies is that the fraction of massive $\left(M>10^{11} M_{\odot}\right)$ LIRGs and ULIRGs at $z<1$ is very small, implying that these objects do not significantly contribute to the total SFRD over this redshift range. Since the majority of the LIRGs and ULIRGs at $0.5<z<1$ are associated with intermediate-mass systems, the absence of these galaxies from our sample is likely to lead to an underestimation of the contribution from these objects (i.e., intermediate-mass systems) to the global SFRD. Moreover, Le Floc'h et al. (2005) found that at $z>0.6$ dusty starbursts with SFR $>10 M_{\odot} \mathrm{yr}^{-1}$ dominate the cosmic star-formation density, with their contribution increasing from $50 \%$ at $z \sim 0$ to $80 \%$ at $z \sim 1$ (i.e., only $20 \%$ of the SFRD at $z \sim 1$ is due to the UV bright galaxies). Any selection bias against these galaxies may possibly compromise our estimate of the SFRD in the range $0.6<z<1.2$. We believe that a possible bias against such galaxies does not affect our results here. By estimating the SFRDs through integrating the rest-frame UV (2800 $\AA$ ) LFs (corrected for dust obscuration) over their entire magnitude range, we incorporate a contribution from galaxies at the faint end of the UV LF, where heavily obscured starbursts would lie. The excellent agreement between our estimated SFRDs from COSMOS and those based on the sample compiled by Hopkins $\&$ Beacom (2006) in Figure 4 supports the fact that the bias against dusty starburst galaxies does not significantly affect the results in the present study. We note that Hopkins \& Beacom (2006) estimate the total contribution to the SFRD from their compilation by combining the SFRD inferred from the UV and far-IR luminosities. Nevertheless, the limitations imposed by selection criteria and any biases so introduced remain a major source of uncertainty in any such study.

Using a sample of $24 \mu \mathrm{m}$ selected galaxies in GOODS-S, Daddi et al. (2007) found a roughly linear relation between the stellar mass and SFRDs. For a given mass, the SFRD at $z \sim 2$ was larger by factors of $\sim 4$ and $\sim 30$ relative to the star-forming galaxies at $z \sim 1$ and $z \sim 0$, respectively. This is roughly consistent with our results in the two highest-mass bins in Figure 6 . In the range $1<z<2$, the observed $24 \mu \mathrm{m}$ wavelength corresponds to rest-frame $8-12 \mu \mathrm{m}$ bands, sampling the polycyclic aromatic hydrocarbon (PAH) features which are most sensitive to the star-forming activity. The agreement between or results and Daddi et al. (2007) further implies that the potential selection bias against dusty star-forming galaxies in our sample is not significantly affecting the estimated SFRDs.

By dividing our sample into three spectral types, ellipticals, spirals and starbursts, we study the evolution (with redshift) of the SFRD-mass relation for each type. A striking feature, presented in Figure 7, is that the slope of the SFRD $-z$ relations, found for different spectral types of galaxies are strong functions of the stellar mass. We find the massive galaxies, which are dominated by early types, to significantly contribute to the total SFRD at earlier epochs $(z \sim 1)$. This is expected as more-massive galaxies are able to support higher rates of star formation than lower-mass systems, all else being equal. For the intermediate-mass systems, the contribution from early-type galaxies to the global SFRD is minimal, with no significant change with redshift. The relation for lower-mass systems, which are dominated by spirals and starbursts, show a progressive build up of the stellar mass (i.e., increasing SFRD) with cosmic time. The results here imply that the stellar mass of galaxies plays a crucial role in determining their star-formation activity and spectral types and governs the contribution of individual galaxies to the total SFRD.

\section{SUMMARY AND CONCLUSION}

Using multiwavelength data from the COSMOS survey, we studied the dependence of the cosmic star-formation density on redshift, stellar mass, and spectral types. We explored the main parameters which govern the star-formation process in galaxies. Our main results are summarized as follows.

1. There is a strong dependence of the SFRD on stellar mass of galaxies, with the most-massive systems $\left(\log \left(M / M_{\odot}\right)>\right.$ 10.5 ) contributing least (by a factor of $\sim 5$ ) to the cosmic SFRD at any given redshift.

2. Combining data from the COSMOS and GDDS, we extend the SFRD $-z-$ mass relation to $z \sim 2$. For high-mass galaxies, we find a steep increase in this relation till $z \sim 2$. This implies that the massive galaxies seen today went through intensive star-formation activity at $z>1$, to generate their current stellar mass. For the less-massive 
systems, the SFRD $-z$ relation flattens at $z \sim 1$, indicating that these systems are currently undergoing build up of their mass, with most of their stellar mass formed at lower redshifts $(z<1)$.

3. We study the dependence of the SFRD- $z-$ mass relation on the spectral type of galaxies. We find that the slope of the SFRD $-z$ relation for different spectral types is a strong function of their stellar mass. For low- and intermediatemass systems, the main contribution to the cosmic SFR comes from the star-forming galaxies while, for moremassive galaxies, older and redder galaxies are the most dominant population, with their contribution to the global SFRD increasing with redshift.

\section{REFERENCES}

Abraham, R., et al. 2007, in Proc. IAU Symp. 235, in Galaxy Evolution Across the Hubble Time, ed. F. Combes \& J. Palou (Dordrecht: Kluwer)

Afonso, J., Hopkins, A., Mobasher, B., \& Almeida, C. 2003, ApJ, 597, 269

Baldry, I. K., \& Glazebrook, K. 2003, ApJ, 593, 258

Bolzonella, M., Miralles, J.-M., \& Pelló, R. 2000, A\&A, 363, 476

Brinchmann, J., Charlot, S., White, S. D. M., Tremonti, C., Kauffmann, G., Heckman, T., \& Brinkmann, J. 2004, MNRAS, 351, 1151

Bruzual, G., \& Charlot, S. 2003, MNRAS, 344, 1000

Calzetti, D., et al. 2007, ApJ, 666, 870

Calzetti, D., Armus, L., Bohlin, R. C., Kinney, A. L., Koornneef, J., \& StorchiBergmann, T. 2000, ApJ, 533, 682

Capak, P., et al. 2007a, ApJS, 172, 99

Capak, P., et al. 2007b, ApJS, 172, 284

Caputi, K. I., Dunlop, J. S., McLure, R. J., \& Roche, N. D. 2005, MNRAS, 361, 607

Caputi, K. I., McLure, R. J., Dunlop, J. S., Cirasuolo, M., \& Schael, A. M. 2006, MNRAS, 366, 609

Coleman, G. D., Wu, C.-C., \& Weedman, D. W. 1980, ApJS, 43, 393

Cowie, L. L., Songaila, A., Hu, E. M., \& Cohen, J. G. 1996, AJ, 112,839
Cram, L., Hopkins, A., Mobasher, B., \& Rowan-Robinson, M. 1998, ApJ, 507, 155

Daddi, E., et al. 2007, arXiv:astro-ph/07052831

Dahlen, T., Mobasher, B., Dickinson, M., Ferguson, H. C., Giavalisco, M., Kretchmer, C., \& Ravindranath, S. 2007, ApJ, 654, 172

Dickinson, M., et al. 2004, ApJ, 600, L99

Ellison, et al. 2007, arXiv:astro-ph/07114833

Flores, H., et al. 1999, ApJ, 517, 148

Giavalisco, M., et al. 2004, ApJ, 600, L103

Glazebrook, K., et al. 2005, in Proc. IAU Symp. 216, Maps of the Cosmos, ed. M. Colless, L. Staveley-Smith, \& R. Stathakis (Dordrecht: Kluwer)

Gómez, P. L., et al. 2003, ApJ, 584, 210

Heavens, A., Panter, B., Jimenez, R., \& Dunlop, J. 2004, Nature, 428, 625

Hopkins, A. M. 2004, ApJ, 615, 209

Hopkins, A. M., \& Beacom, J. F. 2006, ApJ, 651, 142

Hopkins, A. M., Connolly, A. J., Haarsma, D. B., \& Cram, L. E. 2001, AJ, 122, 288

Hopkins, A. M., et al. 2003, ApJ, 599, 971

Juneau, S., et al. 2005, ApJ, 619, L135

Kaufmann, T., Wheeler, C., \& Bullock, J. S. 2007, arXiv:astro-ph/7060210

Kennicutt, R. C. 1998, ARA\&A, 36, 189

Kinney, A. L., Calzetti, D., Bohlin, R. C., McQuade, K., Storchi-Bergmann, T., \& Schmitt, H. R. 1996, ApJ, 467, 38

Le Floc'h, E., et al. 2005, ApJ, 632, 169

Li, C., Kauffmann, G., Heckman, T., Jing, Y. P., \& White, S. D. M. 2007, arXiv:astro-ph/07113792

Mobasher, B., et al. 2007, ApJS, 172, 117

Panter, B., Jimenez, R., Heavens, A., \& Charlot, S. 2007, MNRAS, 378, 1550

Papovich, C., et al. 2006, ApJ, 640, 92

Salvato, M., et al. 2008, arXiv:0809.2098

Scarlata, C., et al. 2007, ApJS, 172, 406

Scoville, N. Z., et al. 2007a, ApJS, 172, 1

Scoville, N. Z., et al. 2007b, ApJS, 172, 150

Seymour, N., et al. 2008, MNRAS, 386, 1695

Strolger, L.-G., et al. 2004, ApJ, 613, 200

Sullivan, M., Mobasher, B., Chan, B., Cram, L., Ellis, R., Treyer, M., \& Hopkins, A. 2001, ApJ, 558, 72

Taniguchi, Y., et al. 2007, ApJS, 172, 9

Zheng, X. Z., Bell, E. F., Papovich, C., Wolf, C., Meisenheimer, K., Rix, H.-W., Rieke, G. H., \& Somerville, R. 2007, ApJ, 661, L41 